\title{
c-myc Inhibition of MyoD and Myogenin-Initiated Myogenic Differentiation
}

\author{
JEFFREY H. MINER AND BARBARA J. WOLD* \\ Division of Biology 156-29, California Institute of Technology, Pasadena, California 91125
}

Received 3 December 1990/Accepted 19 February 1991

\begin{abstract}
In vertebrate development, a prominent feature of several cell lineages is the coupling of cell cycle regulation with terminal differentiation. We have investigated the basis of this relationship in the skeletal muscle lineage by studying the effects of the proliferation-associated regulator, c-myc, on the differentiation of MyoD-initiated myoblasts. Transient cotransfection assays in NIH 3T3 cells using MyoD and c-myc expression vectors demonstrated c-myc suppression of MyoD-initiated differentiation. A stable cell system was also developed in which MyoD expression was constitutive, while myc levels could be elevated conditionally. Induction of this conditional c-myc suppressed myogenesis effectively, even in the presence of MyoD. c-myc suppression also prevented up-regulation of a relative of $\mathrm{MyoD}$, myogenin, which is normally expressed at the onset of differentiation in all muscle cell lines examined and may be essential for differentiation. Additional experiments tested whether failure to differentiate in the presence of myc could be overcome by providing myogenin ectopically. Cotransfection of c-myc with myogenin, MyoD, or a mixture of myogenin and MyoD showed that neither myogenin alone nor myogenin plus MyoD together could bypass the c-myc block. The efifects of c-myc were further dissected by showing that c-myc can inhibit differentiation independently of Id, a negative regulator of muscle differentiation. These results lead us to propose that c-myc and Id constitute independent negative regulators of muscle differentiation, while myogenin and any of the other three related myogenic factors (MyoD, Myf-5, and MRF4/herculin/Myf-6) act as positive regulators.
\end{abstract}

During skeletal muscle development, multipotential precursor cells progress through a determined myoblast stage before differentiating to become myocytes. While precursors and myoblasts proliferate extensively, withdrawal from the cell cycle is required for differentiation (13). The onset of differentiation is characterized by the expression of genes that code for muscle-specific enzymes, contractile proteins, and receptors that are not expressed in myoblasts (reviewed in reference 48). The determination step that establishes a cell as a myoblast can be forced in many fibroblast cell lines by expression of any one of the four members of the MyoD family of myogenic regulatory genes. This family (reviewed in reference 42) includes genes encoding $\operatorname{MyoD}(17,54)$, myogenin $(20,59)$, and Myf-5 (7) and the rat gene encoding MRF4 (47) which is called herculin in mice (37) and Myf-6 in humans (6). All share a highly similar 57-amino-acid B-HLH domain characterized by a strongly basic region in the amino portion followed by a potential helix-loop-helix (HLH) structure (40). Similar domains are also found in an extended family of nuclear proteins (reviewed in reference 31 ). In vertebrates, these include the $m y c$ family of protooncogenes (reviewed in reference 14), twist (28), achaete-scute (29), and the E12 and E47 proteins, first identified as binding to the immunoglobulin $\kappa$-chain enhancer (40).

In vitro studies of DNA binding and protein-protein interactions, together with mutagenesis studies, show that the B-HLH domain is functionally important $(9,16,34,41)$. The HLH portion mediates protein-protein interactions between molecules to yield homo- or hetero-oligomers. Dimers or higher order oligomers are the molecular species capable of binding to DNA in a sequence-specific fashion, and such binding is dependent on the presence of an intact basic region in each monomer. Some mutants with mutations in

\footnotetext{
* Corresponding author.
}

the basic region of MyoD which do bind DNA in vitro do not activate muscle-specific gene expression in vivo, suggesting that the basic region plays a complex role in gene activation (16).

Further support for the roles of the basic and HLH regions in producing active DNA-binding proteins comes from the nature of the protein Id (2). Id contains an HLH domain but not an adjacent basic region. It can associate in vitro with MyoD, E12, and E47 and inhibit their binding to DNA. In vivo, overexpression of Id inhibits the trans-activation of the muscle creatine kinase (MCK) enhancer upon cotransfection with MyoD. Thus, Id, which is widely expressed, appears to negatively regulate the differentiation functions of tissuespecific B-HLH complexes by sequestering components of such complexes in inactive hetero-oligomers (2).

The structural similarity of the MyoD family of regulators with the myc family of proto-oncogenes is interesting because c-myc is strongly implicated in the control of cell proliferation in several different cell lineages, including muscle $(30,51)$. During myogenesis, c-myc is expressed in proliferating myoblasts, but myc mRNA and protein levels drop substantially when these cells withdraw from the cell cycle and differentiate $(22,51) . m y c$ is further implicated in the proliferation-differentiation switch by the fact that differentiation-defective derivatives of some myoblast cell lines fail to down-regulate c-myc expression (51). Other studies show that ectopic myc expression can interfere with myoblast differentiation $(18,24,50)$. Conversely, MyoD has recently been shown to inhibit the serum-induced progression of quiescent cells into $S$ phase $(15 a, 52)$. Considered together, the pattern of myc expression, its sequence similarity to MyoD, its nuclear localization, and the apparent effects of ectopic expression make attractive the possibility that $m y c$ and MyoD play pivotal and opposing roles in controlling the differentiation decision.

In this study we show that c-myc can inhibit the differen- 
tiation of cells made myogenic by transfection of a gene encoding MyoD in both transient and stable assays of NIH $3 T 3$ cells. An important virtue of this system is the ability to study the activities of ectopic MyoD, myogenin, and c-myc in the absence of Myf-5, herculin, and endogenous MyoD, as these genes are not activated in these cells by $\operatorname{MyoD}(37,55)$. Our results show that MyoD and myogenin are not sufficient, acting alone or together, to drive muscle differentiation in the presence of high levels of c-myc. Moreover, the myc suppression can function independently of Id activity, suggesting that there are at least two negative regulators normally expressed in undifferentiated myoblasts that can prevent differentiation.

\section{MATERIALS AND METHODS}

Expression vector constructions. The MT-myc gene in 3T3 MT-myc cells contains the metallothionein-I promoter (EcoRI to BglII, [38]) fused to a c-myc minigene containing the last 33 nucleotides (nt) of the first intron (from the $X b a I$ site), exons 2 and 3 (without intron 2) up to the $N$ siI site (3), and the metallothionein-I polyadenylation signal sequence. The MyoD expression vehicle pEMc11s was a gift from Andrew Lassar (17). Simian virus 40 (SV40)-promoted genes used in the transient cotransfections were all constructed in pECE (21), a 2.9-kb vector which contains the SV40 early promoter and $\mathrm{T}$ antigen polyadenylation signal sequences flanking a multiple cloning site. pSVc11s contains the 1.8-kb EcoRI fragment (MyoD cDNA) from pEMc1ls cloned into the EcoRI site of pECE. pSVmyc contains the 1.4-kb $X b a I-t o-X h o I$ fragment of c-myc (from MT-myc) cloned into the $X b a I$ site of pECE via a three-step ligate-blunt-ligate procedure. pSVrGEN contains rat myogenin cDNA (a gift from Woody Wright) as a 1.5-kb EcoRI fragment from BS-11, which contains the cDNA (59), cloned into the EcoRI site of pECE. Standard procedures were used for all manipulations (36).

Cell culture and DNA transfections. NIH 3T3 cells obtained from the American Type Culture Collection were grown in Dulbecco's Modified Eagle's Medium (DMEM) (Flow)-10\% calf serum (GIBCO or Irvine Scientific) supplemented with penicillin $(10 \mathrm{U} / \mathrm{ml})$ and streptomycin $(10 \mu \mathrm{g} / \mathrm{ml})$ (Irvine Scientific). 3T3 MT-myc cells were grown in DMEM$10 \%$ fetal bovine serum (FBS) (HyClone). Prior to stable transfection with the MyoD expression vehicle $\mathrm{pEMc11s,}$ NIH 3T3 cells and 3T3 MT-myc cells were plated on 10-cm-diameter gelatin-coated plates in DMEM-10\% FBS. They were transfected by calcium phosphate coprecipitation (57) with $10 \mu \mathrm{g}$ of pEMc11s-1 $\mu \mathrm{g}$ of $\mathrm{pY} 3$ (which confers resistance to hygromycin B [4])-5 $\mu \mathrm{g}$ of sheared L cell DNA (as carrier). After $12 \mathrm{~h}$, cells were treated with $15 \%$ glycerol in DMEM for $2 \mathrm{~min}$, rinsed with phosphate-buffered saline, and fed DMEM-15\% FBS. Twenty-four hours later the medium was supplemented with $200 \mu \mathrm{g}$ of hygromycin B per $\mathrm{ml}$ (Calbiochem). After 10 days, surviving colonies were fed differentiation medium (DMEM, 2\% horse serum [Flow Labs], and $8 \mu \mathrm{g}$ of insulin per ml [GIBCO]). Colonies which began to show morphological changes characteristic of myocytes were picked after 36 to $48 \mathrm{~h}$ and expanded in DMEM-15\% FBS.

NIH 3T3 cells used in transient transfections were plated on uncoated dishes in DMEM-10\% calf serum. Transfections were performed as described above, but the glycerol shock was omitted. Plasmid mixtures are described in the figure legends. Forty-eight hours after transfection, plates were fed DMEM-2\% horse serum-2 $\mu \mathrm{g}$ of insulin per $\mathrm{ml}$ to induce differentiation. Three days later plates were fixed and immunostained for the myosin heavy chain (MHC) with horseradish peroxidase as described by Rhodes and Konieczny (47) by using the monoclonal antibody MF20 (1) and Amersham secondary reagents.

RNA isolation and analysis. RNAs were prepared by the method of Chomczynski and Sacchi (12) from cells on $15-\mathrm{cm}$-diameter dishes by using $4 \mathrm{ml}$ of guanidinium solution. Relevant transcripts were analyzed by ribonuclease protection assays. Hybridizations were done in $30 \mu \mathrm{l}$ of $80 \%$ deionized formamide- $40 \mathrm{mM}$ PIPES (pH 6.4) [piperazine$N, N^{\prime}$-bis(2-ethanesulfonic acid)]-1 mM EDTA-400 mM $\mathrm{NaCl}$ under mineral oil at $50^{\circ} \mathrm{C}$ for $12 \mathrm{~h}$ by using 2.5 or $5 \mu \mathrm{g}$ of total RNA and $5 \times 10^{9}$ radiolabeled probe molecules per hybridization. Unhybridized probes were digested by adding $300 \mu \mathrm{l}$ of $10 \mathrm{mM}$ Tris (pH 7.5)-5 mM EDTA-300 mM NaCl-1 $\mathrm{U}$ of RNase T1 (Bethesda Research Laboratories) per $\mu$ l. In addition, $0.36 \mathrm{ng}$ of RNase A (Sigma) per ml was used for human $\gamma$-actin and Id probe digestions. Ribonucleases were digested with proteinase K (Boehringer Mannheim), samples were extracted with phenol-chloroform-isoamyl alcohol (25: 24:1), and protected probes were isopropanol precipitated with $30 \mu \mathrm{g}$ of yeast type III RNA (Sigma) as a carrier. Pellets were dissolved in $80 \%$ formamide and electrophoresed through $8 \%$ denaturing polyacrylamide gels, which were exposed to Kodak XAR-5 film at $-70^{\circ} \mathrm{C}$ with an intensifying screen.

Probes for ribonuclease protections. Radiolabeled riboprobes were synthesized by using SP6 (Promega), T3, or T7 (Pharmacia or US Biochemical Corp.) RNA polymerase and $\left[\alpha{ }^{32}\right.$ P]CTP or UTP (Amersham or New England Nuclear). pSP6 $g$-actin contains a fragment of the human $\gamma$-actin gene (26) in pSP64. When cut with HinfI and transcribed with SP6 RNA polymerase, the resulting 145 -nt probe protects a 65 -nt fragment of mouse $\gamma$-actin RNA (23). The MyoD probe BH8 contains the $\sim 600$-bp HindIII-to-BamHI fragment of pEMc11s inserted into Bluescript $\mathrm{KS}^{+}$(Stratagene). BH8 is cut with StyI (nt 1692 in reference 17) and transcribed with T7 RNA polymerase to make a probe that produces a 116-nt protected fragment from transcripts off pEMc11s and a 92-nt fragment from transcripts off the endogenous MyoD gene. The mouse myc probe used extends from the EcoRV site in the second exon to the $X b a I$ site in the first intron and was transcribed with T3 RNA polymerase. This probe distinguishes between transcripts from the MT-myc gene and the endogenous gene. The mouse myogenin plasmid SRmyo8 contains the 224-bp EcoRIto-SmaI fragment ( 5 ' end in reference 20 ) inserted into EcoRIand SmaI-cut Bluescript II KS ${ }^{+}$. This plasmid is cut with StyI (nt 35 of myogenin) and transcribed with T7 RNA polymerase. The Id plasmid pMH18 R (2) was cut with DdeI at nt 817 and transcribed with T7 RNA polymerase to make a 174-nt probe, $110 \mathrm{nt}$ of which is protected by Id RNA.

\section{RESULTS}

myc inhibits differentiation initiated by MyoD in transient cotransfection assays. To test the effects of c-myc on MyoDinitiated myogenesis, we performed a series of transient cotransfections in which the absolute amount of MyoD was held constant and the amount of myc was varied. To avoid difficulties stemming from differences in promoter strength, both genes were placed under the control of the constitutive SV40 early promoter in the plasmid pECE (21). Also, to guard against artifactual effects due to competition for factors driving the SV40 promoter, these transfection mixtures 


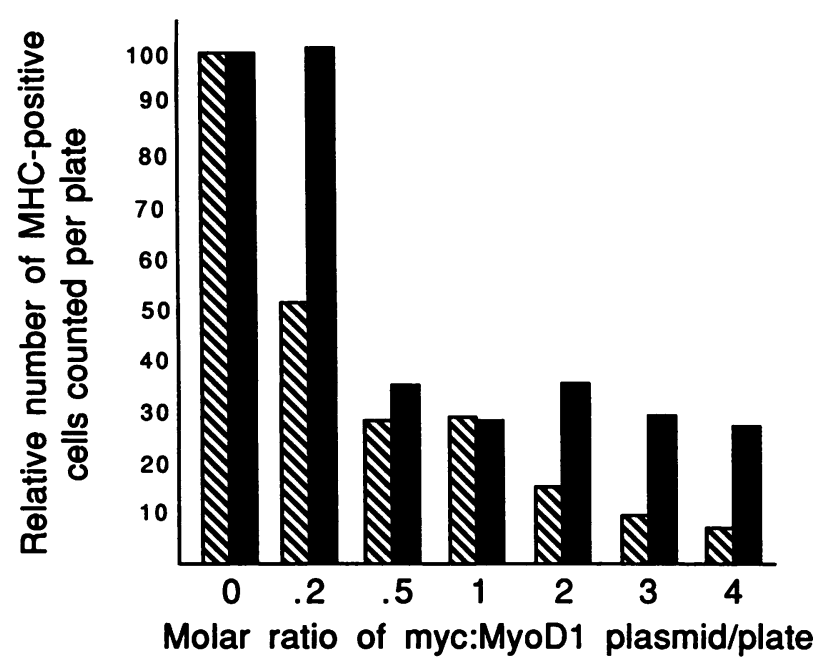

FIG. 1. Transient inhibition of MyoD-mediated myogenesis by c-myc. These experiments show that in transient cotransfections, differentiation is most efficient at low molar ratios of myc to MyoD plasmids and is least efficient at higher ratios. NIH 3T3 cells were transfected in two separate experiments (represented by hatched and black bars) using different myc plasmid preparations. DNA mixtures contained $5 \mu \mathrm{g}$ of pSVcl1s (SV40 early promoter-driven $\mathrm{MyoD})$, enough of the myc plasmid pSVmyc (SV40 early promoterdriven $m y c$ ) to make the indicated $m y c /$ MyoD ratios, enough pECE (SV40 promoter alone) to equalize the molar amount of SV40 promoter per plate, and either $1 \mu \mathrm{g}$ of pY3, $1.5 \mu \mathrm{g}$ of pSV2CAT, plus enough sheared $L$ cell DNA to make $25.8 \mu \mathrm{g}$ of DNA per $10-\mathrm{cm}$ plate (for hatched bars) or $2 \mu \mathrm{g}$ of pSV2CAT plus enough pT7T3-18 (Bethesda Research Laboratories) to make $25.3 \mu \mathrm{g}$ of DNA per plate (for black bars). Chloramphenicol acetyltransferase activity was not significantly variable from point to point (data not shown). For the experiment represented by the hatched bars, 20 fields at $25 \times$ power on each of two plates were counted and averaged per point; 100 represents 1,960 MHC-positive cells. For the experiment represented by the black bars, 25 fields on one plate were counted per point; 100 represents 12,722 MHC-positive cells.

all contained the requisite amount of $\mathrm{pECE}$ vector such that the total molar amount of SV40 promoter in each transfection mixture was identical. NIH 3T3 cells were transfected with these mixtures along with the SV40-promoted bacterial chloramphenicol acetyltransferase gene (pSV2CAT) to control for significant transfection efficiency differences from plate to plate. After 3 to 4 days in differentiation medium, the cells were fixed and the number of MHC-positive cells were counted after immunostaining. The results of two such experiments (hatched and black bars) are shown in Fig. 1. As MyoD is held constant and c-myc is increased, the number of differentiated muscle cells diminishes by up to $90 \%$. These results suggest that $c-m y c$ has a strong inhibitory effect on the ability of MyoD to promote differentiation in NIH 3T3 cells. By comparison with prior observations of retroviral inhibition of primary myoblast differentiation (24) or transfections of naturally occurring myoblast cell lines (e.g., reference 18) in which the complement of muscle regulators already being expressed is unknown, the results in this system specifically show inhibition of MyoD-initiated differentiation.

myc overexpression inhibits MyoD-initiated differentiation in stable transfectants. There are likely to be important intermediate steps between expression of ectopic MyoD and activation of muscle-specific genes. Otherwise, any cell which expresses MyoD would become myogenic; but this is not the case (56). However, while the transient assays show that c-myc inhibits MyoD-initiated myogenesis, the small fraction of cells which actually take up and express DNA are not suitable for further characterization of the regulatory pathway. We therefore constructed a set of NIH 3T3-based cell lines that are myogenic because of constitutive expression of a transfected MyoD cDNA. Some of these lines also carry a c-myc gene under the control of the mouse metallothionein-I promoter (MT-myc gene) which can be induced up to 10-fold above endogenous myc protein levels by supplementing the culture medium with zinc sulfate (54a). However, these MT-myc cells do not appear to be growth transformed, as they do not form foci in culture or tumors in nude mice, even when treated with zinc. A useful feature of conditional myc expression is that it helps to ensure that the phenotypic effects observed are attributable to transient overexpression of c-myc and not to long-term secondary effects of chronic myc expression or to the emergence of differentiation-defective derivatives, a possible factor in prior studies of the effects of myc on myogenesis. Myogenic derivatives of these MT-myc cells and of the parental NIH 3T3 cells were made in parallel transfections by using the MyoD expression vector $\mathrm{pEMc11s}$ (17). The myogenic potential of MyoD-transfected cell clones was evaluated by immunostaining for expression of MHC after cultivation for several days in a mitogen-poor differentiation medium. Several independent myogenic transformants in both 3T3 and 3T3 MT-myc backgrounds were subcloned, and the most myogenic of these were chosen for further study.

MyoD myoblasts carrying the zinc-inducible MT-myc gene (MT-myc/MyoD1 cells) differentiate in the absence of added metal, but differentiation is suppressed in the presence of zinc. MyoD myoblasts lacking the metal-inducible myc gene (MyoD1 cells) are unaffected by zinc and differentiate to similar extents in the presence or absence of added metal. This result is visualized by the immunocytochemical stain for MHC shown in Fig. 2A, and a quantitation is presented in Fig. 2B. Differentiated cells were scored by counting cells that stain positive for MHC; similar results have been obtained by immunostaining for MCK and by quantitating MCK RNA levels (data not shown). Differentiation was not completely suppressed when myc was induced, a result which is expected if the quantity of c-myc expressed is crucial for suppression, since immunostaining for c-myc has shown that it does not accumulate to uniformly high steady-state levels in all cells of the MT-myc line (54a). We conclude that in these cells, differentiation is specifically suppressed by the induction of MT-myc expression.

myc suppresses differentiation even in the presence of MyoD. How are the differentiation phenotypes in these experiments related to the levels of MyoD and myc expression? To begin to answer this question, the levels of myc and MyoD transcripts were measured by a ribonuclease protection assay. Results shown in Fig. 3 confirm that in the MT- $m y c /$ MyoD line, $m y c$ transcripts from the transgene accumulate to significantly elevated levels in the presence of added zinc (Fig. 3; in the 3.5-h exposure of the myc probe, compare lane 1 with 2 and lane 3 with 4 and in the longer exposure, compare lane 5 with 6). Lower myc levels are observed in the absence of added zinc when cells differentiated well, while the elevated myc levels are from cells that failed to differentiate efficiently (as in Fig. 2). It is relevant that by the criteria of appearance, adherence to the plate, yield of RNA, and level of expression of cytoplasmic $\boldsymbol{\gamma}$-actin RNA (Fig. 3), all cells seemed equally healthy under all 


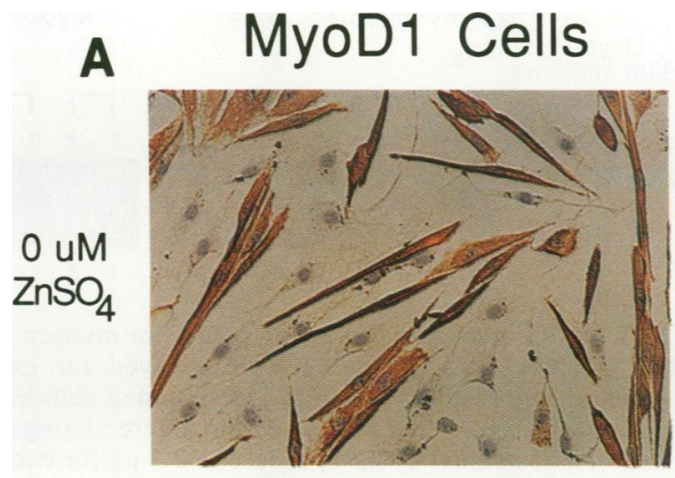

\section{MT-myc/MyoD1 Cells}
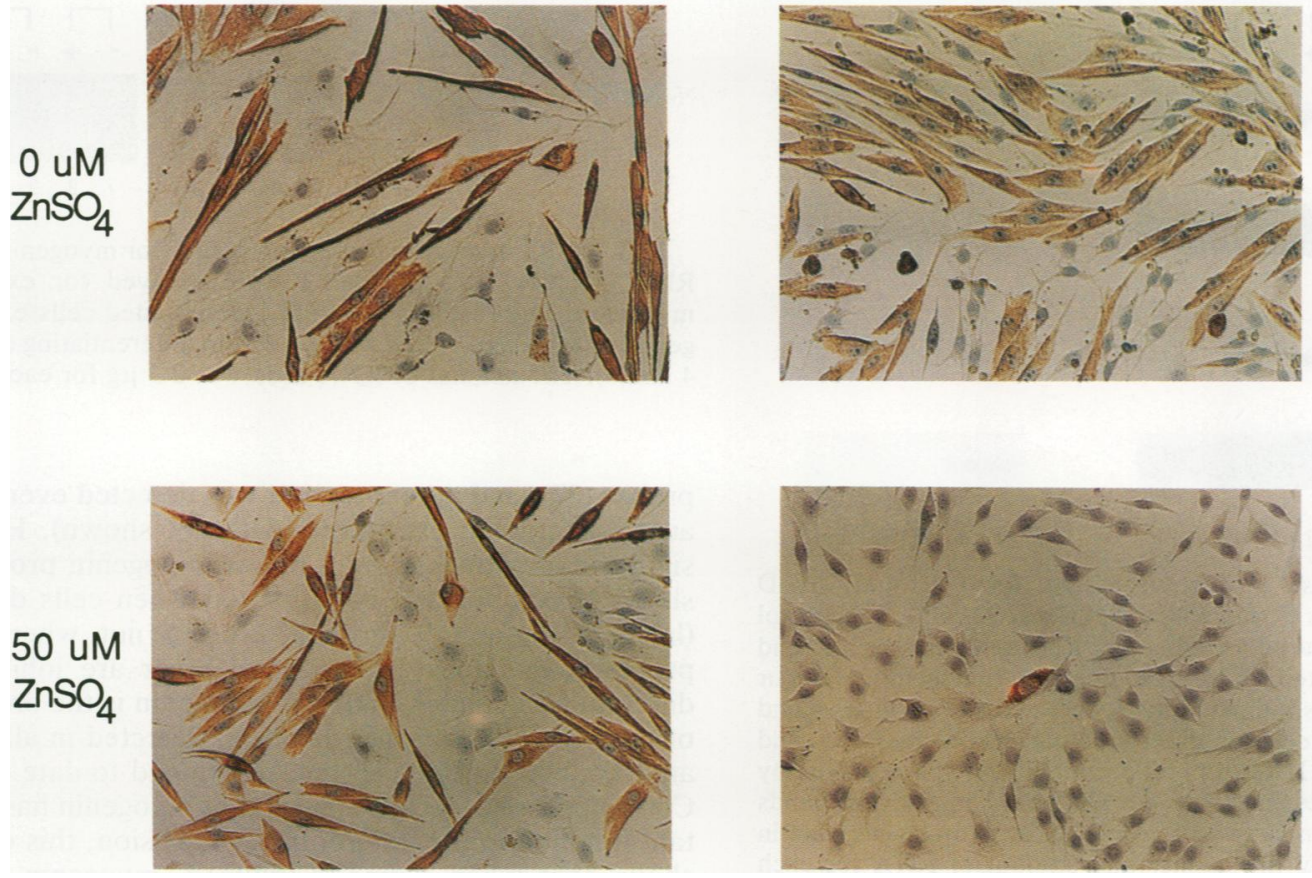

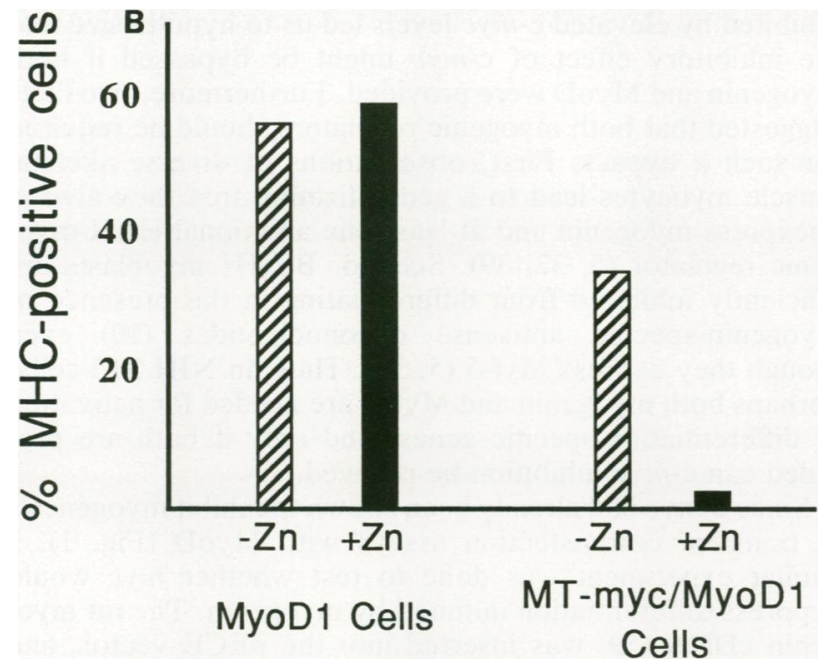

conditions tested. Also, changes in myc RNA levels in 3T3 and MT-myc 3T3 cells have been found to reflect similar changes in myc protein levels (54a), although it remains possible that there is some additional control at the translational level for myc or for MyoD (54) in these cells.

Levels of MyoD expression were measured, and they revealed that under all conditions transgene MyoD was expressed. MyoD1 control cells expressed lower levels of MyoD than did MT-myc/MyoD1 cells, yet both cell lines differentiated well (unless $m y c$ was elevated). The probe used to measure MyoD discriminated between the transgene (shown in Fig. 3) and endogenous MyoD. In both cell lines
FIG. 2. Muscle differentiation suppressed by zinc-dependent induction of c-myc in MT-myc/MyoD1 cells but not in control MyoD1 cells. (A) Immunocytochemical staining for the presence of muscle MHC in MyoD1 cells and in MT-myc/MyoD1 cells. Differentiated cells stain brown, while cells that do not express MHC are counterstained light violet with hematoxylin. Cells were cultured for 6 days in a mitogen-poor differentiation medium (DMEM containing $1 \%$ horse serum and $5 \mu \mathrm{g}$ of insulin per $\mathrm{ml}$ ) with the concentration of zinc sulfate indicated. Zinc induces expression of myc from the MT-myc transgene in 3T3 MT-myc cells. Note that cell fusion does not occur in differentiated MyoD1 or MT-myc/MyoD1 cells. (B) Quantitation of an experiment similar to that described for panel A. Cells were cultured in differentiation medium with $50 \mu \mathrm{M}$ (black bars) or no (hatched bars) zinc sulfate for $\sim 6$ days. Note that differentiation is suppressed in zinc-treated MT-myc cells, while zinc has no inhibitory effect on differentiation of MyoD1 cells lacking the MT-myc gene. Additionally, zinc had no significant effect on the average number of cells present in the random microscopic fields which were assayed.

the endogenous MyoD gene was silent (37) and therefore made no contribution to overall MyoD levels. In the MTmyc/MyoD1 line, levels of MyoD decreased modestly at later time points in the presence of metal (Fig. 3; for the MyoD probe, compare lane 3 with 4 and lane 5 with 6). However, this decrease is not likely to be the direct cause for inhibition of differentiation, because even the reduced MyoD levels substantially exceed amounts that are sufficient for differentiation in the control MyoD1 cell line (Fig. 3, lanes 7 through 12).

These data, taken together with the levels of myc expressed in MT-myc/MyoD1 cells and control MyoD1 cells, 


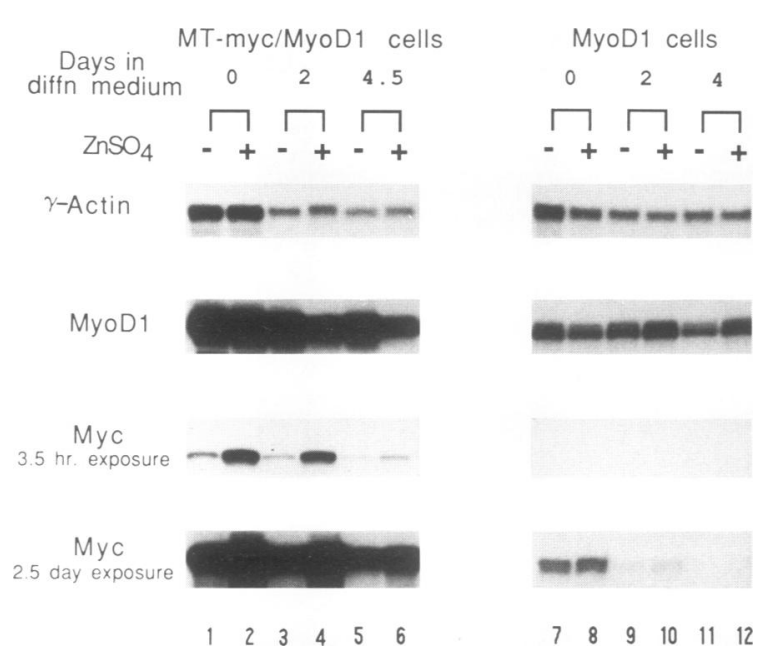

FIG. 3. Ribonuclease protection assays for $m y c$ and MyoD RNAs. Total RNA was extracted from MT-myc/MyoD1 and control MyoD1 cells were cultured as indicated. RNAs in lanes 1, 2, 7, and 8 are from cells harvested $3 \mathrm{~h}$ after feeding with rich medium with or without zinc sulfate (as indicated by + or - ). RNAs in lanes 4 and 6 are from cells inhibited from differentiating, while lanes 3,5, and 9 through 12 contain RNAs from differentiated cells, as assayed by MHC and MCK expression (data not shown). The relevant bands have been excised from large gels. A probe for cytoplasmic $\gamma$-actin (23) was used to verify the quality and amount of RNA for each timepoint, which was $2.5 \mu \mathrm{g}$ (determined spectrophotometrically). Note the two different exposures for myc as indicated.

place some informative constraints on how c-myc and MyoD might be acting on the differentiation decision. Comparison of the absolute amount of total c-myc RNA in each of the two cell lines in the absence of zinc (Fig. 3, lanes 1, 3, and 5 compared with lanes 7,9 , and 11 , respectively, of the 2.5-day exposure of the myc probe) shows that the two express significantly different levels. c-myc levels that are permissive for differentiation in MT-myc/MyoD1 cells exceed those expressed in rapidly proliferating MyoD1 cells (e.g., lane 7) and other myoblast cell lines (data not shown). Nevertheless, when the MT-myc gene is induced with zinc, myc levels rise high enough-perhaps over a threshold-to suppress differentiation even in the presence of abundant amounts of MyoD (Fig. 3, lanes 4 and 6). In this context, the observed decrease in MyoD RNA levels from lane 3 to 4 and lane 5 to 6 associated with added zinc may serve to lower any threshold over which c-myc levels must rise to efficiently suppress MyoD-dependent differentiation. Therefore, we conclude that c-myc effects on myogenesis depend on the quantity of c-myc, not its mere presence or absence. The amount required for suppression of differentiation in these cells is high, exceeding levels that characterize myoblasts prior to differentiation. However, these cells also express high levels of MyoD, suggesting that the ratio of c-myc to myogenic HLH family gene products is the crucial parameter.

Myogenin is expressed in differentiated cells but not in MT-myc/MyoD1 cells inhibited from differentiating. Mechanisms that may rely on quantitative relationships among HLH molecules must also accommodate additional family members that may ultimately participate in MyoD-initiated myogenesis. To further characterize the repertoire of $\mathrm{HLH}$ muscle regulators in these cells, we performed RNase protection experiments by using mouse Myf-5 and herculin
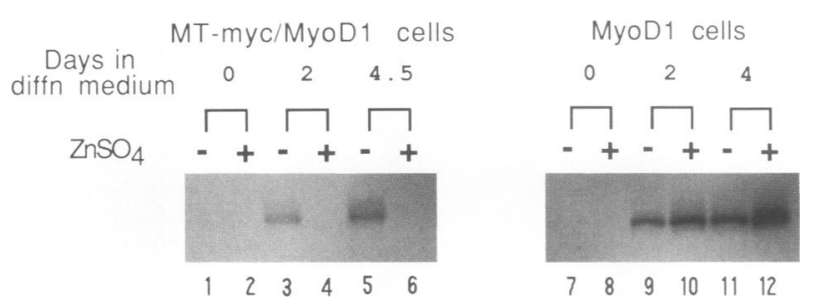

FIG. 4. Ribonuclease protection assays for myogenin RNA. The RNA samples used in Fig. 3 were assayed for expression of myogenin. The results show that differentiated cells express myogenin, but those inhibited by c-myc from differentiating do not (lanes 4 and 6). The amount of RNA used was $2.5 \mu \mathrm{g}$ for each lane.

probes (37), and no expression was detected even after long autoradiographic exposures (data not shown). However, a similar experiment using a mouse myogenin probe (Fig. 4) shows that myogenin is expressed when cells differentiate (lanes 3, 5, and 9 through 12) but not when cells are proliferating (lanes 1, 2, 7, and 8) or are inhibited from differentiating (lanes 4 and 6). Myogenin is the only member of the MyoD family that has been detected in all differentiated skeletal muscle cell lines examined to date $(5,37,39)$. Consistent with the hypothesis that myogenin has an important function in the differentiation decision, this experiment shows that when c-myc is induced, myogenin expression fails to be activated.

Can myogenin or MyoD-myogenin coexpression bypass the inhibitory effects of $m y c$ ? The observation that up-regulation of myogenin and subsequent myogenic differentiation are inhibited by elevated c-myc levels led us to hypothesize that the inhibitory effect of c-myc might be bypassed if both myogenin and MyoD were provided. Furthermore, two facts suggested that both myogenic regulators should be required for such a bypass. First, observations of diverse skeletal muscle myocytes lead to a generalization that they always coexpress myogenin and at least one additional HLH myogenic regulator $(5,37,39)$. Second, $\mathrm{BC}_{3} \mathrm{H} 1$ myoblasts are efficiently inhibited from differentiating in the presence of myogenin-specific antisense oligonucleotides (10) even though they express Myf-5 $(5,39)$. Thus, in NIH 3T3 cells, perhaps both myogenin and MyoD are needed for activation of differentiation-specific genes, and only if both are provided can c-myc inhibition be relieved.

Since c-myc had already been shown to inhibit myogenesis in transient cotransfection assays with MyoD (Fig. 1), a similar experiment was done to test whether myc would suppress differentiation initiated by myogenin. The rat myogenin cDNA (59) was inserted into the pECE vector, and NIH 3T3 cells were transfected with the mixtures indicated in Fig. 5. The amount of myc plasmid used was one-half the total molar amount of myogenic plasmid. After 3 days in differentiation medium, cells were fixed and immunostained for MHC, and differentiated cells were counted. The results show that MyoD and myogenin act cooperatively when transfected together, in that they convert significantly more NIH 3T3 cells to myocytes than do the same molar doses of myogenin or MyoD alone (cf., reference 49). Nevertheless, c-myc inhibited differentiation whether MyoD, myogenin, or a mixture of the two was used to initiate myogenesis. Thus, although the activation of myogenin that normally accompanies differentiation of MyoD1 cells is suppressed by c-myc, myogenin provided ectopically cannot relieve the overall inhibition of differentiation. The straightforward interpreta- 


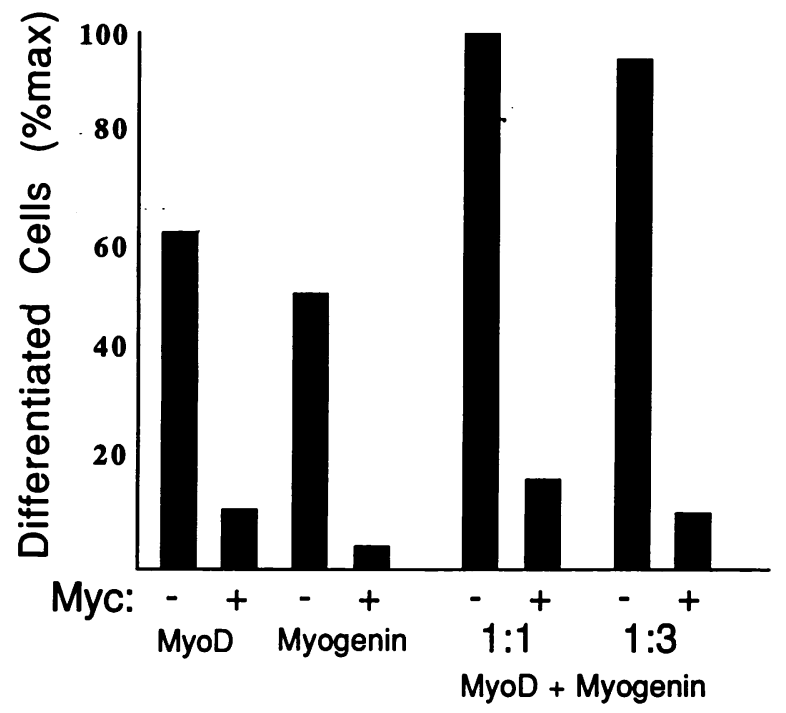

Transfection Mixture

FIG. 5. Transient cotransfection of myogenin, alone or with MyoD, cannot bypass c-myc inhibition of myogenesis. NIH 3T3 cells were plated on 6-cm-diameter dishes. Each transfection mixture was divided among three dishes and contained the molar equivalent of $20 \mu \mathrm{g}$ of pSVclls as its myogenic component. Note that the total molar amount of myogenic plasmid in each mixture of MyoD and myogenin is equal to their molar amounts when used individually. pSVmyc $(9.1 \mu \mathrm{g})$ was sometimes included, as indicated, which was half that molar amount. Otherwise, $6.2 \mu \mathrm{g}$ of pECE was included to balance the total SV40 promoter content. The transfection procedure was as described in Materials and Methods, except that $70 \mu \mathrm{M}$ chloroquine was added to the medium during the first $6 \mathrm{~h}$ of transfection, after which the cells were rinsed with DMEM, fed fresh medium, and then switched to differentiation medium $24 \mathrm{~h}$ later for 3 days. After fixing and staining for MHC, positive cells in 15 fields were counted at a $25 \times$ magnification. In this experiment, the maximum was 670 differentiated cells counted. Two repetitions of this experiment yielded similar qualitative results.

tion is that in these cells, MyoD and myogenin together may be necessary for differentiation, but they are not sufficient to force differentiation in the presence of c-myc.

Expression of Id is not influenced by ectopic c-myc levels and is down-regulated even in cells inhibited from differentiating. The pattern of expression of Id, a negative regulator of MyoD-initiated muscle gene transcription (2), parallels the pattern of endogenous c-myc expression during myogenesis. Both are expressed at relatively high levels during proliferation compared with those expressed upon differentiation. This raised the possibility of a hierarchical relationship between the two in which elevated c-myc would exert its phenotypic effect by driving expression of Id. Thus, in our studies, myc might inhibit differentiation indirectly by positively regulating expression of Id in zinc-induced MT-myc/ MyoD1 cells under mitogen-poor conditions, whereas Id levels would be down-regulated in the absence of zinc because of lower levels of c-myc. However, a direct experimental test of this possibility found Id to be down-regulated identically in the presence and absence of suppressing levels of c-myc. Figure 6 shows that even in cells that are inhibited from differentiating by c-myc (lanes 4 and 6), Id levels are no higher than those in cells that have differentiated (lanes 3, 5, and 9 through 12). Thus, while c-myc and Id are normally
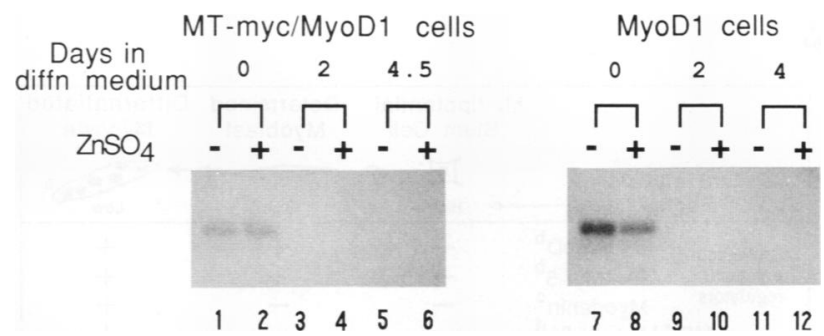

FIG. 6. Ribonuclease protection assays for Id RNA. The RNA samples from Fig. 3 were assayed for expression of Id, an inhibitor of differentiation. The results show that Id expression is properly down-regulated even in cells exhibiting c-myc-dependent suppression of differentiation (lanes 4 and 6). The amount of RNA used was $5 \mu \mathrm{g}$ for each lane.

similar in their expression patterns under growth factor-rich and -poor conditions (Fig. 7A), we have detected no alteration in Id levels in response to ectopic c-myc.

\section{DISCUSSION}

c-myc is normally expressed in proliferating myoblasts and is down-regulated upon differentiation into myocytes $(22,51)$. We have begun to dissect the regulatory effects of c-myc on myogenesis initiated by MyoD, myogenin, or a combination of the two. In these experiments, expression of c-myc has been uncoupled from environmental and cellular factors which normally influence its regulation. The central result is that preventing the drop in c-myc expression that normally occurs at the onset of differentiation results in inhibition of muscle differentiation.

Comparison of $m y c$-mediated inhibition with effects of other negative regulators. Our experiments suggest a significant role for $c-m y c$ in the differentiation decision which differs from the roles of other negative regulators tested in previous studies. Earlier steps in myogenesis are affected by perturbations in ras $(32,35,43,44,53)$, proliferin (58), c-fos (35), and perhaps src (24), all leading to suppression of muscle differentiation, though there are conflicting results in some systems (27). Mutant-activated ras and excess fos have been shown to prevent or reduce transcription of the endogenous MyoD gene $(32,35)$, and in one study activated ras-dependent inhibition of myogenic differentiation was shown to be accompanied by an up-regulation of c-myc RNA, even in differentiation medium (43). Proliferin appears to act by reducing the level of $\mathrm{MyoD}$ expression or by altering the MyoD transcript, perhaps making it nonfunctional as MyoD mRNA (58). In contrast to these examples of myogenic inhibition, which act on regulation of MyoD, we have shown that c-myc is able to inhibit differentiation in cell lines at a more distal point in the regulatory pathway-even in the presence of constitutive MyoD. However, while our use of the NIH 3T3 cell line afforded experimental control of MyoD levels in the absence of endogenous MyoD expression, it prevented us from determining whether $c-m y c$, like activated ras and fos, can down-regulate an active endogenous MyoD gene. A further observation in our study is that the c-myc inhibition of cellular differentiation cannot be overcome by expression of myogenin, alone or in conjunction with MyoD. By contrast, in the case of activated ras or fos, constitutive MyoD from a stably transfected cDNA bypassed their suppressing effects $(32,35)$.

While the present study shows that c-myc inhibits myogenic differentiation effectively in both transient and stable 
A

\begin{tabular}{|c|c|c|c|c|}
\hline \multicolumn{2}{|c|}{$\begin{array}{l}\text { Culture Medium } \\
\text { Growth Factor Level } \longrightarrow\end{array}$} & $\begin{array}{l}\text { Multipotential } \\
\text { Stem Cell } \\
\rightarrow \text { Hog }\end{array}$ & $\begin{array}{c}\begin{array}{c}\text { Determined } \\
\text { Myoblast }\end{array} \\
\text { Hoh }\end{array}$ & $\rightarrow \underbrace{\substack{\text { Differentiated } \\
\text { Myocyte }}}_{\text {Low }}$ \\
\hline $\begin{array}{l}\text { positive-acting } \\
\text { myogenic } \\
\text { regulators } \\
\text { MRF }\end{array}$ & $\begin{array}{r}\text { MyoD b } \\
\text { Myf- } 5^{b} \\
\text { Myogeninc } \\
\text { 4/ Herculind }\end{array}$ & $\begin{array}{l}- \\
- \\
-\end{array}$ & $\begin{array}{l}+ \\
+ \\
-\end{array}$ & $\begin{array}{l}+ \\
+ \\
+ \\
+\end{array}$ \\
\hline coregulators & E12/E47 & + & + & + \\
\hline $\begin{array}{l}\text { negative-acting } \\
\text { regulators }\end{array}$ & $\begin{array}{r}\text { c-myc } \\
\text { Id }\end{array}$ & $\begin{array}{l}+ \\
+\end{array}$ & $\begin{array}{l}+ \\
+\end{array}$ & - \\
\hline $\begin{array}{l}\text { mature muscle } \\
\text { markers }\end{array}$ & $\begin{array}{r}\text { MHC } \\
\text { MCK } \\
\alpha \text {-actins }\end{array}$ & $\bar{z}$ & $\bar{z}$ & $\begin{array}{l}+ \\
+\end{array}$ \\
\hline
\end{tabular}

B

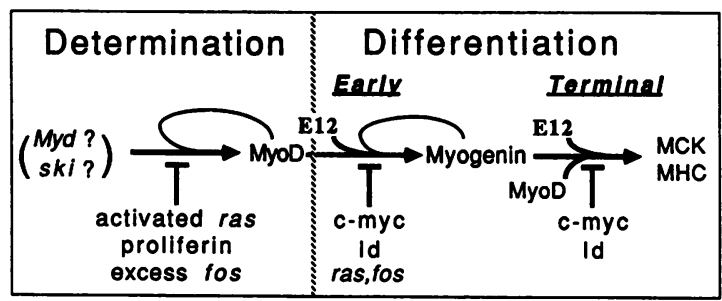

FIG. 7. (A) Expression patterns of genes involved in myogenic determination and differentiation in cultured cell lines. The data summarize expression patterns in naturally occurring skeletal muscle cell lines; ectopic expression of any one of the four MyoD family myogenic regulators by transfection can change these patterns. References: MyoD (17), myogenin (20, 59), Myf-5 (5, 39), MRF4/ herculin (37, 47), E12/E47 (40), c-myc (51), Id (2), MHC, MCK, and $\alpha$-actins (48). ${ }^{a}$ While many cell lines fuse into multinucleated myotubes concurrent with biochemical differentiation, $\mathrm{BC}_{3} \mathrm{H} 1$ cells do not; however, transfection of a MyoD expression vector into these cells corrects the fusion defect (8). ${ }^{b}$ While we find that differentiated $\mathrm{C} 2 \mathrm{C} 12$ cells express all four myogenic regulators, many cell lines express only myogenin and either MyoD (e.g., MM14 cells) or Myf-5 (e.g., $\mathrm{BC}_{3} \mathrm{H} 1$ cells) but not both. ${ }^{c}$ Although we have detected myogenin RNA in nominally proliferating MM14 and $\mathrm{C} 2 \mathrm{C} 12$ cells $(37,39)$, this is presumed to be contributed by spontaneously differentiated cells, which are frequently found in those cultures even in the presence of high levels of growth factors. ${ }^{d}$ We detect herculin in differentiated $\mathrm{C} 2 \mathrm{C} 12$ cells (37) and at much lower levels in both proliferating and differentiated $\mathrm{BC}_{3} \mathrm{H} 1$ cells (36a); low level expression of MRF4 (the herculin homolog in rats) has been reported in differentiated L6 cells (47) and in L8 cells (6). Otherwise, while herculin is abundantly expressed in adult mouse skeletal muscle tissue, it is rarely expressed in cultured cell lines. However, myf-6 (the herculin homolog in humans) is expressed in primary human myocytes in culture (6). (B) A speculative model for a myogenic regulatory network. Arrows represent activation, loops represent autoregulation, bars represent inhibition, and curves represent cooperative activation. E12 is meant to be representative of the several known daughterless homologs which can heterooligomerize with the MyoD family members.

transfection formats, the situation for ras and fos is more complicated. Their inhibitory effects can be bypassed by stable transfection with a MyoD expression vector, yet they cannot be bypassed when transiently cotransfected with MyoD and a muscle-specific chloramphenicol acetyltransferase reporter gene into $\mathrm{C} 3 \mathrm{H} 10 \mathrm{~T} 1 / 2$ cells (35). In that report
(35), it was suggested that the negative activities of ras and fos dominate over the positive activities of MyoD in a transient assay but not in stable cell lines because the former may allow for a higher ratio of oncogene-to-MyoD activity. In addition, it is not certain what, if any, contribution is made to total MyoD levels by the endogenous gene in the transients (cf., reference 54), nor is it known whether myogenin is expressed or whether c-myc is up-regulated (as in reference 43 ). Whatever the explanation for the differences observed in the two assays, the results show that activated ras or excess fos may also act at a step later than MyoD expression, as c-myc clearly does.

Mechanisms of c-myc action. What are the possible mechanisms for c-myc inhibition of muscle differentiation? c-myc is localized in nuclei and contains motifs characteristic of sequence-specific DNA-binding proteins, including the B-HLH motif, essential for oligomerization and function of MyoD family regulators $(16,41)$, and a leucine zipper, a motif known to function in dimerization of other transcription factors such as fos and jun (33). These characteristics suggest two direct mechanisms for myogenic inhibition. In one, myc would bind to promoters or enhancers of downstream muscle-specific genes and act as a repressor. An alternative direct mechanism would have myc inactivate positive-acting muscle-specific transcription factors of the MyoD type by binding to them or to an essential limiting partner. Indeed, the apparent sensitivity of c-myc inhibition to the myc-to-MyoD ratio (shown in this study) is consistent with such a titration by c-myc. However, attempts to achieve dimerization of $\mathrm{c}-m y c$ with these (or other) potential partners in standard in vitro assays have thus far failed (31). Moreover, strong evidence supporting sequence-specific DNA binding for myc is also lacking, though myc does have a general affinity for DNA $(19,45)$. Nevertheless, the initial negative results of such in vitro studies do not convincingly rule out the mechanisms outlined above.

Less direct mechanisms to explain c-myc effects on differentiation must also be considered. c-myc was first identified as an oncogene, thereby implicating it in the control of cell proliferation. More recent studies assign c-myc a significant role in regulating entry into $G_{1}$ and $S$ phases of the cell cycle (11). This is relevant to skeletal myogenesis because it is well established that the permissive point in the cell cycle for the onset of differentiation is the $G_{0}-G_{1}$ boundary (13). Thus, since c-myc can drive a subset of quiescent NIH 3 T3 cells out of $G_{0}$ and into $G_{1}$ and $S$ phases, its ability to inhibit differentiation may be secondary to its ability to activate genes involved in progression through the cell cycle. Arguing against this view are two observations. (i) Up to $75 \%$ of MT-myc/MyoD1 cells differentiated in some of our experiments, and the extent of inhibition by c-myc induction exceeded $80 \%$, thus involving a far larger fraction of MTmyc cells than can ever be forced into $\mathrm{S}$ phase by a myc induction, which is $\sim 30 \%$ (36b). (ii) Although progression into $\mathrm{S}$ phase does not appear to fully explain the observed phenotype, a myc-mediated progression into a position in $\mathrm{G}_{1}$ which is prohibitive for differentiation is possible. While definitive studies will require isolation and use of markers that subdivide progress into and through $G_{1}$, our data showing a lack of Id expression characteristic of cycling cells (Fig. 6) also argue against a mechanism dependent on cycling.

Positive and negative regulatory pathways of myogenesis in cultured cell lines. Figure 7A summarizes the expression patterns of positively and negatively acting myogenic regulatory genes in cell lines in culture and includes the expres- 
sion pattern of some mature muscle markers. These data provide the biological context for a simple speculative model based upon the observations in this paper together with prior studies (Fig. 7B). It accommodates the effects of the positive- and negative-acting regulators presently known and integrates data from naturally occurring myogenic cell lines as well as transfection-initiated myoblast lines. The first step identified is determination, the transition from a multipotential stem cell to a myoblast. Here it is accomplished by expression of MyoD, although in other instances Myf-5 might substitute at this step. As indicated, MyoD is sometimes (but not always) able to establish an autoregulatory loop (55). The initial trigger of MyoD expression is presently unknown, but one plausible candidate is the genetically defined myd locus, which has been shown to activate MyoD when transfected into nonmyogenic cells (46). The ski oncogene, which can also convert fibroblasts to myoblasts in transfection assays (15), may also be involved. Negative regulators that act by disrupting MyoD expression include activated ras, excess c-fos, and proliferin, and they appear to be able to function even after the MyoD autoregulatory loop has been established $(32,35,58)$. c-myc and Id have not yet been formally tested for a similar negative effect on MyoD expression in proliferating myoblasts. However, under growth factor-rich conditions that support proliferation of MyoD-positive myoblasts, significant levels of both c-myc and Id have been found in all cases, and we therefore think it is very likely that their expression is compatible with expression of MyoD.

At the next step in the pathway, MyoD-positive myoblasts are signalled to differentiate, which is accomplished in vitro by removing serum growth factors. A rapid response to reduced growth factors is a drop in Id and c-myc expression. One expected result will be the release of any B-HLH proteins that were previously nonfunctional because of sequestration by Id through protein-protein interactions. Indeed, E12 and MyoD both bind efficiently to Id in vitro, and this association inactivates their DNA-binding capacities. These observations led Benezra et al. (2) to propose that a MyoD/E12 hetero-oligomer is necessary for the onset of differentiation, and this activation step is indicated as the early phase of differentiation in Fig. 7B. Within the resolution of our kinetic measurements, myogenin expression begins concurrent with the drop in Id and c-myc; myogenin then appears to be able to positively autoregulate $(5,20)$. Experiments presented here and by Benezra et al. (2) indicate that this kinetically early step in differentiation (relative to MHC and MCK expression) is inhibited by c-myc and Id. Additionally, Lassar et al. (35) provide evidence that high levels of mutant ras or fos relative to MyoD also inhibit the myogenin activation step, and the results of Olson et al. (43) suggest that such inhibition by activated ras may actually be mediated by up-regulation of c-myc expression. Since myogenin has been found to be expressed in all differentiating myogenic cell lines examined $(5,37,39)$, this step may be essential for differentiation. Direct support for this comes from studies which show that $\mathrm{BC}_{3} \mathrm{H} 1$ and $\mathrm{L6}-\mathrm{A} 1$ myoblasts are efficiently inhibited from differentiating in the presence of myogenin-specific antisense oligonucleotides $(10,25)$.

As differentiation proceeds, muscle-specific genes such as those encoding MHC and MCK are transcribed, and in some cases, cell fusion occurs to produce terminally differentiated myotubes. As E12 can dimerize with myogenin (9) as well as with MyoD $(2,41)$ and can bind to essential cis-acting elements in muscle-specific enhancers, both myogenin-E12 and MyoD-E12 complexes are good candidates for playing a central role in transcriptional activation of terminal differentiation genes. This final step in Fig. 7B is responsive to inhibition by ectopic c-myc expression, as demonstrated in our cotransfections using c-myc, myogenin, and MyoD. Id has not yet been formally tested for its ability to inhibit similarly in the presence of MyoD and myogenin. However, the molecular data showing that Id can inactivate E12 in vitro (2) suggest that ectopic Id expression will also block the final step of differentiation.

The group of positive and negative myogenic regulatory factors presented in Fig. 7 have been identified as such primarily by testing their activities in cultured cell lines and in in vitro biochemical studies. The repertoire of regulators described thus far is probably incomplete, but among those currently known some redundancy in both positive and negative regulators is already apparent. For example, all four MyoD family regulators can convert some nonmyogenic cells to myoblasts, yet some cell lines differentiate efficiently without expression of Myf-5 or herculin, while others can proceed without MyoD. Additionally, our results suggest that c-myc and Id can act independently as negative regulators. This leads to the prediction that even if Id expression is eliminated in myoblasts cultured in growth factor-rich medium, such myoblasts will not spontaneously differentiate because c-myc would provide an independent negative control (unless Id positively regulates c-myc activity-a testable possibility). Conversely, elimination of c-myc expression should not allow for differentiation if Id expression is maintained. Therefore, the minimal requirement for premature activation of muscle-specific genes in myoblasts would be the inactivation of both c-myc and Id.

The existence of at least two independent negative regulators of muscle differentiation may be useful to the organism, because such redundancy would ensure that differentiation does not occur before myoblasts have migrated to their proper locations in the developing embryo and have proliferated sufficiently to properly fill their compartments. Because of its positive effects on progression through the cell cycle and its negative effects on differentiation, c-myc appears well suited as a physiological regulator that simultaneously prevents premature differentiation and promotes expansion of precursor cell numbers in lineages where linkage of these activities is important.

\section{ACKNOWLEDGMENTS}

We are indebted to A. Lassar, R. Davis, and H. Weintraub for the MyoD cDNA and helpful suggestions; to $R$. Benezra and $H$. Weintraub for the Id cDNA; to W. Wright and E. Olson for rat and mouse myogenin cDNAs, respectively; to $\mathrm{S}$. Tavtigian for the MT-myc cell line; to S. Hauschka and J. Johnson for immune reagents and MM14 and C2C12 cell lines; to R. Miake-Lye for the pECE vector; and to $K$. Lee for help with cell counting. We also thank D. Anderson, N. Davidson, P. Sternberg, and members of the Wold group for helpful insights during the course of this work and for comments on the manuscript.

J.H.M. was supported by a National Science Foundation Graduate Fellowship and by National Research Service Award GM07616 from the National Institute of General Medical Sciences. This work was supported by grants to B.W. from the National Institutes of Health, the Rita Allen Foundation, and the Muscular Dystrophy Association.

\section{REFERENCES}

1. Bader, D., T. Masaki, and D. A. Fischman. 1982. Immunochemical analysis of myosin heavy chain during avian myogenesis in vivo and in vitro. J. Cell Biol. 95:763-770.

2. Benezra, R., R. L. Davis, D. Lockshon, D. L. Turner, and $H$. 
Weintraub. 1990. The protein Id: a negative regulator of helixloop-helix DNA binding proteins. Cell 61:49-59.

3. Bernard, O., S. Cory, S. Gerondakis, E. Webb, and J. M. Adams. 1983. Sequence of the murine and human cellular myc oncogenes and two models of myc transcription resulting from chromosomal translocation in B lymphoid tumors. EMBO J. 2:2375-2383.

4. Blochlinger, K., and H. Diggelmann. 1984. Hygromycin B phosphotransferase as a selectable marker for DNA transfer experiments with higher eucaryotic cells. Mol. Cell. Biol. 4:29292931.

5. Braun, T., E. Bober, G. Buschhausen-Denker, S. Kotz, K. H. Grzeschik, and H. H. Arnold. 1989. Differential expression of myogenic determination genes in muscle cells: possible autoactivation by the $M y f$ gene products. EMBO J. 8:3617-3625.

6. Braun, T., E. Bober, B. Winter, N. Rosenthat, and H. H. Arnold. 1990. Myf-6, a new member of the human gene family of myogenic determination factors: evidence for a gene cluster on chromosome 12. EMBO J. 9:821-831.

7. Braun, T., G. Buschhausen-Denker, E. Bober, E. Tannich, and H. H. Arnold. 1989. A novel human muscle factor related to but distinct from MyoD1 induces myogenic conversion in 10T1/2 fibroblasts. EMBO J. 8:701-709.

8. Brennan, T. J., D. G. Edmondson, and E. N. Olson. 1990. Aberrant regulation of MyoD1 contributes to the partially defective myogenic phenotype of $\mathrm{BC}_{3} \mathrm{H} 1$ cells. J. Cell Biol. 110:929-937.

9. Brennan, T. J., and E. N. Olson. 1990. Myogenin resides in the nucleus and acquires high affinity for a conserved enhancer element on heterodimerization. Genes Dev. 4:582-595.

10. Brunetti, A., and I. D. Goldfine. 1990. Role of myogenin in myoblast differentiation and its regulation by fibroblast growth factor. J. Biol. Chem. 265:5960-5963.

11. Cavalieri, F., and M. Goldfarb. 1987. Growth factor-deprived BALB/c 3T3 murine fibroblasts can enter the $S$ phase after induction of c-myc gene expression. Mol. Cell. Biol. 7:3554 3560.

12. Chomczynski, P., and N. Sacchi. 1987. Single-step method of RNA isolation by acid guanidinium thiocyanate-phenol-chloroform extraction. Anal. Biochem. 162:156-159.

13. Clegg, C. H., T. A. Linkhart, B. B. Olwin, and S. D. Hauschka. 1987. Growth factor control of skeletal muscle differentiation occurs in $\mathrm{G}_{1}$-phase and is repressed by fibroblast growth factor. J. Cell Biol. 105:949-956.

14. Cole, M. D. 1986. The myc oncogene: its role in transformation and differentiation. Annu. Rev. Genet. 13:361-384.

15. Colmenares, C., and E. Stavnezer. 1989. The ski oncogene induces muscle differentiation in quail embryo cells. Cell 59: 293-303.

15a.Crescenzi, M., T. P. Fleming, A. B. Lassar, H. Weintraub, and S. A. Aaronson. 1990. MyoD induces growth arrest independent of differentiation in normal and transformed cells. Proc. Natl. Acad. Sci. USA 87:8442-8446.

16. Davis, R. L., P.-F. Cheng, A. B. Lassar, and H. Weintraub. 1990. The MyoD DNA binding domain contains a recognition code for muscle-specific gene activation. Cell 60:733-746.

17. Davis, R. L., H. Weintraub, and A. B. Lassar. 1987. Expression of a single transfected $\mathrm{cDNA}$ converts fibroblasts to myoblasts. Cell 51:987-1000.

18. Denis, N., S. Blanc, M. P. Leibovitch, N. Nicolaiew, F. Dautry, M. Raymondjean, J. Kruh, and A. Kitzis. 1987. c-myc oncogene expression inhibits the initiation of myogenic differentiation. Exp. Cell Res. 172:212-217.

19. Donner, P., I. Greiser-Wilke, and K. Moelling. 1982. Nuclear localization and DNA binding of the transforming gene product of avian myelocytomatosis virus. Nature (London) 296:262-266.

20. Edmondson, D. G., and E. N. Olson. 1989. A gene with homology to the myc similarity region of MyoD1 is expressed during myogenesis and is sufficient to activate the muscle differentiation program. Genes Dev. 3:628-640.

21. Ellis, L., E. Clauser, D. O. Morgan, M. Edery, R. A. Roth, and W. J. Rutter. 1986. Replacement of insulin receptor tyrosine residues 1162 and 1163 compromises insulin-stimulated kinase activity and uptake of 2-deoxyglucose. Cell 45:721-732.

22. Endo, T., and B. Nadal-Ginard. 1986. Transcriptional and posttranscriptional control of c-myc during myogenesis: its mRNA remains inducible in differentiated cells and does not suppress the differentiated phenotype. Mol. Cell. Biol. 6:14121421.

23. Enoch, T., K. Zinn, and T. Maniatis. 1986. Activation of the human $\beta$-interferon gene requires an interferon-inducible factor. Mol. Cell. Biol. 6:801-810.

24. Falcone, G., F. Tato, and S. Alema. 1985. Distinctive effects of the viral oncogenes $m y c$, erb, fps, and $s r c$ on the differentiation program of quail myogenic cells. Proc. Natl. Acad. Sci. USA 82:426-430.

25. Florini, J. R., and D. Z. Ewton. 1990. Highly specific inhibition of IGF-I-stimulated differentiation by an antisense oligodeoxyribonucleotide to myogenin mRNA. J. Biol. Chem. 265:1343513437.

26. Gunning, P., P. Ponte, H. Okayama, J. Engel, H. Blau, and L. H. Kedes. 1983. Isolation and characterization of full-length cDNA clones for human $\alpha-, \beta-$, and $\gamma$-actin mRNAs: skeletal but not cytoplasmic actins have an amino-terminal cysteine that is subsequently removed. Mol. Cell. Biol. 3:787-795.

27. Harrington, M. A., F. Gonzales, and P. A. Jones. 1988. Effect of cellular determination on oncogenic transformation by chemicals and oncogenes. Mol. Cell. Biol. 8:4322-4327.

28. Hopwood, N. D., A. Pluck, and J. B. Gurdon. 1989. A Xenopus mRNA related to Drosophila twist is expressed in response to induction in the mesoderm and the neural crest. Cell 59:893903.

29. Johnson, J. E., S. J. Birren, and D. J. Anderson. 1990. Two rat homologues of Drosophila achaete-scute are specifically expressed in neuronal precursors. Nature (London) 346:858-861.

30. Kelly, K., B. H. Cochran, C. D. Stiles, and P. Leder. 1983. Cell-specific regulation of the c-myc gene by lymphocyte mitogens and platelet-derived growth factor. Cell 35:603-610.

31. Kingston, R. E. 1989. Transcription control and differentiation: the HLH family, c-myc and C/EBP. Curr. Opin. Cell Biol. 1:1081-1087.

32. Konieczny, S. F., B. L. Drobes, S. L. Menke, and E. J. Taparowsky. 1989. Inhibition of myogenic differentiation by the $\mathrm{H}$-ras oncogene is associated with the down regulation of the MyoD1 gene. Oncogene 4:473-481.

33. Landschulz, W. H., P. F. Johnson, and S. L. McKnight. 1988. The leucine zipper: a hypothetical structure common to a new class of DNA binding proteins. Science 240:1759-1764.

34. Lassar, A. B., J. N. Buskin, D. Lockshon, R. L. Davis, S. Apone, S. D. Hauschka, and H. Weintraub. 1989. MyoD is a sequencespecific DNA binding protein requiring a region of $m y c$ homology to bind to the muscle creatine kinase enhancer. Cell 58:823-831.

35. Lassar, A. B., M. J. Thayer, R. W. Overell, and H. Weintraub. 1989. Transformation by activated ras or fos prevents myogenesis by inhibiting expression of MyoD1. Cell 58:659-667.

36. Maniatis, T., E. F. Fritsch, and J. Sambrook. 1982. Molecular cloning: a laboratory manual. Cold Spring Harbor Laboratory, Cold Spring Harbor, N.Y.

36a.Miner, J. H. Unpublished data.

36b.Miner, J. H., and S. V. Tavtigian. Unpublished data.

37. Miner, J. H., and B. Wold. 1990 . Herculin, a fourth member of the MyoD family of myogenic regulatory genes. Proc. Natl. Acad. Sci. USA 87:1089-1093.

38. Mueller, P. R., S. J. Salser, and B. Wold. 1988. Constitutive and metal-inducible protein:DNA interactions at the mouse metallothionein I promoter examined by in vivo and in vitro footprinting. Genes Dev. 2:412-427.

39. Mueller, P. R., and B. Wold. 1989. In vivo footprinting of a muscle specific enhancer by ligation mediated PCR. Science 246:780-786.

40. Murre, C., P. Schonleber McCaw, and D. Baltimore. 1989. A new DNA binding and dimerization motif in immunoglobulin enhancer binding, daughterless, MyoD and myc proteins. Cell 56:777-783.

41. Murre, C., P. Schonleber McCaw, H. Vaessin, M. Caudy, L. Y. 
Jan, Y. N. Jan, C. V. Cabrera, J. N. Buskin, S. D. Hauschka, A. B. Lassar, H. Weintraub, and D. Baltimore. 1989. Interactions between heterologous helix-loop-helix proteins generate complexes that bind specifically to a common DNA sequence. Cell 58:537-544.

42. Olson, E. N. 1990 . MyoD family: a paradigm for development? Genes Dev. 4:1454-1461.

43. Olson, E. N., G. Spizz, and M. A. Tainsky. 1987. The oncogenic forms of N-ras or H-ras prevent skeletal myoblast differentiation. Mol. Cell. Biol. 7:2104-2111.

44. Payne, P. A., E. N. Olson, P. Hsiau, R. Roberts, M. B. Perryman, and M. D. Schneider. 1987. An activated c-Ha-ras allele blocks the induction of muscle-specific genes whose expression is contingent on mitogen withdrawal. Proc. Natl. Acad. Sci. USA 84:8956-8960.

45. Persson, H., and P. Leder. 1984. Nuclear localization and DNA binding properties of a protein expressed by human c-myc oncogene. Science 225:718-721.

46. Pinney, D. F., S. H. Pearson-White, S. F. Konieczny, K. E. Latham, and C. P. Emerson, Jr. 1988. Myogenic lineage determination and differentiation: evidence for a regulatory gene pathway. Cell 53:781-793.

47. Rhodes, S. J., and S. F. Konieczny. 1989. Identification of MRF4: a new member of the muscle regulatory factor gene family. Genes Dev. 3:2050-2061.

48. Rosenthal, N. 1989. Muscle cell differentiation. Curr. Opin. Cell Biol. 1:1094-1101.

49. Schafer, B. W., B. T. Blakely, G. J. Darlington, and H. Blau. 1990. Effect of cell history on response to helix-loop-helix family of myogenic regulators. Nature (London) 344:454-458.

50. Schneider, M. D., M. B. Perryman, P. A. Payne, G. Spizz, R. Roberts, and E. N. Olson. 1987. Autonomous expression of c-myc in $\mathrm{BC}_{3} \mathrm{H} 1$ cells partially inhibits but does not prevent myogenic differentiation. Mol. Cell. Biol. 7:1973-1977.

51. Sejersen, T., J. Sumegi, and N. R. Ringertz. 1985. Density- dependent arrest of DNA replication is accompanied by decreased levels of c-myc mRNA in myogenic but not in differentiation-defective myoblasts. J. Cell. Physiol. 125:465-470.

52. Sorrentino, V., R. Pepperkok, R. L. Davis, W. Ansorge, and L. Philipson. 1990. Cell proliferation inhibited by MyoDI independently of myogenic differentiation. Nature (London) 345:813815.

53. Sternberg, E. A., G. Spizz, M. E. Perry, and E. N. Olson. 1989. A ras-dependent pathway abolishes activity of a muscle-specific enhancer upstream from the muscle creatine kinase gene. Mol. Cell. Biol. 9:594-601.

54. Tapscott, S. J., R. L. Davis, M. J. Thayer, P. F. Cheng, H. Weintraub, and A. B. Lassar. 1988. MyoD1: a nuclear phosphoprotein requiring a myc homology region to convert fibroblasts to myoblasts. Science 242:405-411.

54a.Tavtigian, S. V. Unpublished data.

55. Thayer, M. J., S. J. Tapscott, R. L. Davis, W. E. Wright, A. B. Lassar, and $H$. Weintraub. 1989. Positive autoregulation of the myogenic determination gene MyoD1. Cell 58:241-248.

56. Weintraub, H., S. J. Tapscott, R. L. Davis, M. J. Thayer, M. A. Adam, A. B. Lassar, and A. D. Miller. 1989. Activation of muscle-specific genes in pigment, nerve, fat, liver, and fibroblast cell lines by forced expression of MyoD. Proc. Natl. Acad. Sci. USA 86:5434-5438.

57. Wigler, M., A. Pellicer, S. Silverstein, R. Axel, G. Urlaub, and L. Chasin. 1979. DNA-mediated transfer of the adenine phosphoribosyltransferase locus into mammalian cells. Proc. Natl. Acad. Sci. USA 76:1373-1376.

58. Wilder, E. L., and D. I. H. Linzer. 1989. Participation of multiple factors, including proliferin, in the inhibition of myogenic differentiation. Mol. Cell. Biol. 9:430-441.

59. Wright, W. E., D. A. Sassoon, and V. K. Lin. 1989. Myogenin, a factor regulating myogenesis, has a domain homologous to MyoD. Cell 56:607-617. 